\title{
Effect of three different media on serum free culture of donor corneas and isolated human corneal endothelial cells
}

\author{
Jürgen Bednarz, Vladimir Doubilei, Patricia C M Wollnik, Katrin Engelmann
}

Universitäts-

Augenklinik,

Martinistrasse 52,

D-20246 Hamburg,

Germany

J Bednarz

V Doubilei

K Engelmann

AK Barmbek, Augenabteilung, Rübenkamp 148, D-22307 Hamburg, Germany

P C M Wollnik

Department of Ophthalmology, Kazan Medical Academy, Kazan, Russia

V Doubilei

Correspondence to: Dr Jürgen Bednarz, Universitäts-Augenklinik Eppendorf, Martinistrasse 52, 20246 Hamburg, Germany

bednarz@uke.uni-hamburg.de

Accepted for publication 27 June 2001

\begin{abstract}
Background-Removal of bovine serum from organ culture medium is necessary because of the variability in serum composition and the potential risk of infection. Two specific endothelial cell media (F99 and Endothelial-SFM) were compared with the routinely used medium MEM for their use in serum free cultivation of human corneal endothelial cells (HCEC) and donor corneas.

Methods-HCEC were incubated in three test media with or without increasing serum content and a growth assay was performed. Seven pairs of donor corneas were cultured in each of three media for 3 weeks, one cornea with serum supplementation and one without. Endothelial cell density was determined once each week. Trypan blue staining of the endothelium and vital staining of keratocytes was performed after 3 weeks.

Results-All three media promoted proliferation of cultured HCEC when supplemented with serum. Endothelial cell density of donor corneas was comparable after 3 weeks of cultivation in the different media. Only corneas cultured in medium MEM without serum exhibited a higher endothelial cell loss. Trypan blue staining of the endothelium after cultivation revealed the lowest number of damaged cells on corneas cultured in the medium Endothelial-SFM. The highest densities of keratocytes were found in corneas cultured in Endothelial-SFM and the lowest densities occurred after culture in MEM. Conclusion-After incubation in Endothelial-SFM even under serum free conditions corneas were found to be of higher quality with respect to endothelial cell survival, cell membrane integrity, and keratocyte density. This medium may replace MEM, which is routinely used in European eye banks but requires supplementation with serum.

(Br F Ophthalmol 2001;85:1416-1420)
\end{abstract}

The endothelial cell density is the main criterion for the quality of a donor cornea and is known to decrease during organ culture. ${ }^{1-3}$ Therefore efforts were made to avoid endothelial cell loss during organ culture, and even to increase cell density - for example, by supplementation of the culture medium with mitogenic agents. ${ }^{4}$ Barisani-Asenbauer et $a \bar{p}$ did not find any effect of epithelial growth factor
(EGF) or insulin-like growth factor (IGF) on cultured corneas within the first 2 weeks of culture but they described a stabilisation of the endothelial cell pattern during the following 2 weeks.

Methods to isolate and cultivate human corneal endothelial cells have been established. Specification of the medium and medium supplementation led to the definition of culture conditions for these cells. ${ }^{6}$ Moreover, the suitability of cultured human corneal endothelial cells for testing different media and medium components for their application in cornea banking has been demonstrated before. ${ }^{7}$ Our experience in organ culture as well as in the cultivation of human corneal endothelial cells has exposed a certain inconsistency: on one hand a minimal nutritional environment is used for organ culture of donor corneas using minimal essential medium (MEM) and low serum content ${ }^{8}$ while on the other hand highly defined conditions are necessary for cultivation of isolated human corneal endothelial cells. These findings led to the question of whether more enriched medium than MEM should be used for donor cornea preservation and if such a medium could be used without serum supplementation. This would allow the avoidance of the use of bovine serum for human cornea preservation.

In this study we compared the effect of three different media with or without serum supplementation on cultured corneal endothelial cells as well as on donor corneas. MEM is routinely used for cornea preservation in European eye banks. Medium F99, a 1:1 mixture of medium Ham's F12 and Medium 199, has been shown to be an optimised medium for culture of human corneal endothelial cells. ${ }^{9}$ Medium Endothelial-SFM (Life Technologies) has been designed for cultivation of vascular endothelial cells especially under serum free conditions. The composition of this medium is not published, but it is understood not to contain growth factors or animal derived components. The medium Endothelial-SFM, together with medium F99, has been found to be superior for human cornea preservation in comparison with other commercial available media in a previous study. ${ }^{10}$ In this previous study, all the media were supplemented with bovine serum. In the present study we show for the first time that the use of EndotheliumSFM may allow long term storage of human donor corneas under serum free conditions. 
Material and methods

CELL CULTURE

Human corneal endothelial cells of a 91 year old donor were isolated from an organ cultured cornea that had been deemed unsuitable for transplantation purposes, and then cultured as described elsewhere. ${ }^{69}$ These cells were immortalised by transfecting them with pRNS, ${ }^{11}$ a plasmid containing the genes for the SV40 small and large $\mathrm{T}$ antigens. ${ }^{12}$ The immortalised cells were cultured in medium F99 (a 1:1 mixture of Ham's F12 (Life Technologies) and M199 (Life Technologies)) supplemented with $5 \%$ fetal calf serum, $20 \mu \mathrm{g} / \mathrm{ml}$ ascorbic acid (Sigma), $20 \mu \mathrm{g} / \mathrm{ml}$ bovine insulin (Sigma), 2.5 $\mu \mathrm{g} / \mathrm{ml}$ transferrin (Sigma), $0.6 \mathrm{ng} / \mathrm{ml}$ sodium selenite (Sigma), and $10 \mathrm{ng} / \mathrm{ml}$ basic fibroblast growth factor (rh, Seromed). ${ }^{6}$ They were cultivated in $25 \mathrm{~cm}^{2}$ Costar flasks, ${ }^{13}$ which were maintained at $37^{\circ} \mathrm{C}$ in a humidified atmosphere containing $5 \% \mathrm{CO}_{2}$.

\section{PROLIFERATION ASSAY}

The cells were seeded in $2.0 \mathrm{ml}$ of the above mentioned culture medium onto six well culture dishes (2000 cells/well) from Costar. After 24 hours, most of the cells had attached to the bottom of culture dishes and the medium in each set of culture dishes was replaced by one of three different test media (F99, Endothelial-SFM, and MEM). Test media in each culture dish were supplemented with different amount of FCS $(0 \%, 0.05 \%$, $0.1 \%, 0.5 \%, 1 \%, 5 \%)$. The test media were renewed after 4 days and then on the 6 th and the 8th days. After 4 days of cultivation and then every subsequent day the number of cells was estimated in one of the culture dishes from each set.

DETERMINATION OF CELL NUMBER

Cell number was determined as described before. ${ }^{14}$ The cultures were washed with PBS and cells were removed from the culture dish by adding $1 \mathrm{ml}$ of $0.05 \% / 0.02 \%$ trypsin/EDTA solution and exposure at $37^{\circ} \mathrm{C}$ in air supplemented with $5 \% \mathrm{CO}_{2}$ for 8-12 minutes. The obtained cell suspension was diluted in $9 \mathrm{ml}$ of isotonic salt solution and counted using a Coulter Counter ZM (Coulter Electronics GmbH, Krefeld, Germany).

ORGAN CULTURE

The medium effect on organ cultured corneas was analysed using 21 pairs of donor corneas. These corneas were not suitable for keratoplasty owing to low endothelial cell count, high postmortem time, or failure to maintain serological testing of the donor. The corneas of seven donors were incubated in each of three media, one cornea with and the other without $2 \%$ serum supplementation. The donor corneas were fixed in a corneal holding device and cultured in $50 \mathrm{ml}$ tissue culture flasks at $37^{\circ} \mathrm{C}$ in an atmosphere containing $5 \% \mathrm{CO}_{2} .{ }^{15}$ The medium was not changed during the observation period. Mean donor age was 70.7 (SD 10.3 ) years for corneas incubated in medium MEM, 73.1 (7.2) years for corneas incubated in medium F99, and 72.0 (10.5) years for corneas incubated in medium Endothelial-SFM. The postmortem times were 60.9 (35.1) hours (MEM), 60.9 (24.3) hours (F99), and 49.4 (23.9) hours (Endothelial-SFM), respectively.

EVALUATION OF CORNEAL ENDOTHELIAL CELL DENSITY

Every week each cornea was placed in BSS solution, the endothelium was examined by means of phase contrast microscopy (Labovert FS, Leitz, Germany) and photodocumented. Using these pictures, cell numbers within different frames each representing an area of $0.02 \mathrm{~mm}^{2}$ were counted. Subsequently the cornea was transferred to the same medium and further cultivated as described above.

TRYPAN BLUE STAINING

Trypan blue staining was performed as described before. ${ }^{16}$ After 3 weeks of cultivation the cornea was washed in $0.9 \% \mathrm{NaCl}$ and the endothelium was covered with $200 \mu \mathrm{l}$ trypan blue solution $(0,5 \%$ in $0.9 \% \mathrm{NaCl}$ solution, Serva). After 2 minutes the cornea was again washed in $0.9 \% \mathrm{NaCl}$ solution and the endothelium was evaluated under a microscope. A photograph was taken and the area of blue stained endothelium was determined.

ANALYSIS OF KERATOCYTES

Keratocytes were analysed in corneal sections by means of a live dead assay (Molecular Probes) using fluorescent dyes. Before cutting, the corneas were washed extensively in PBS and endothelium and epithelium of the corneas were removed to avoid high fluorescence by intensely stained endothelial or epithelial cells. From each cornea two longitudinal tissue sections of $2 \mathrm{~mm}$ were excised. One of these sections was further transversally cut into three pieces, one representing the anterior stroma, another two representing central and posterior stroma respectively. Directly before staining a mixture of $2.5 \mu \mathrm{l}$ calcein, $\mathrm{AM}$ ( $4 \mathrm{mM}$ in DMSO), and $10 \mu \mathrm{l}$ ethidium homodimer-1 (2 $\mathrm{mM}$ in $1: 4 \mathrm{DMSO} / \mathrm{H}_{2} \mathrm{O}$ ) in $5 \mathrm{ml}$ PBS was prepared. Tissue sections were incubated in this solution for 40 minutes in the dark. Without any washing steps the tissue sections were placed onto the microscope slides and analysed by means of a fluorescence microscope (Leitz, Fluovert FS).

\section{STATISTICS}

Data are given as mean (SD). Differences in mean values among the groups were compared by analysis of variance (ANOVA) using SPSS Base 10.0 software.

\section{Results}

In the first part of this study we analysed the effect of three different basal media on isolated immortalised human corneal endothelial cells in vitro. All three media supported proliferation of cultured human corneal endothelial cells when supplemented with $0.05 \%$ FCS. Further increase of FCS supplementation resulted in a dose dependent stimulation of cell 



Figure 1 Proliferation of isolated human corneal endothelial cells during culture in medium MEM (A), F99 (B), and Endothelial-SFM (C). Cells were seeded on six well plates (2000 cells/well) and cultured using the indicated medium without serum supplementation or in the presence of $0.05 \%, 0.1 \%, 0.5 \%, 1 \%$, or $5 \%$ FCS, respectively. The number of cells was determined after 4, 5, 6, 7, 8, and 11 days of culture. Indicated cell amounts are means (SD) of six independent experiments.

proliferation (Fig 1). The lowest rates of proliferation were observed in medium EndothelialSFM, especially during the first week of culture. Use of media F99 and MEM led to a decrease in cell number after 1 week (Fig 1) and total cell death was observed after 2-3 weeks. Endothelial-SFM was the only medium that enabled prolonged cultivation without serum supplementation.

The influence of the different media on organ cultured corneas was investigated in the second part of this study. Endothelial cell density decreased during culture in all three media supplemented with FCS or not (Table 1). The highest decrease in cell density occurred within the first week. The further decrease observed during the following 2 weeks was less pronounced. No differences between the media were observed, except in the case of serum free MEM. During culture in this medium the main endothelial cell loss significantly increased (Fig 2). This was due to extended necrotic areas present in the endothelium of four out of seven corneas cultured in medium MEM without FCS after 3 weeks. In medium MEM with $2 \%$ FCS or medium F99 (plus or minus 2\% FCS) comparable endothelial necrotic areas were seen in only one out of seven corneas. Such an extended necrosis was observed in none of the corneas cultured in serum free or serum supplemented Endothelial-SFM medium. A comparable result was observed after trypan blue staining of the corneal endothelium (Fig 3). Nearly half (44\%) of the corneal endothelial cells exhibited positive staining after 3 weeks of cultivation in medium MEM. A reduction of the trypan blue stained area to about $22 \%$ of the corneal surface was observed in serum supplemented MEM. The corresponding values were $13 \%$ for the corneas cultured in F99 and $6 \%$ for corneas cultured in Endothelial-SFM, regardless of serum supplementation.

Statistical analysis revealed that culturing the corneas in SFM led to significant smaller necrotic areas in comparison with MEM. This significant result was observed between both media supplemented or unsupplemented with FCS $(p<0.05)$. However, only serum free conditions showed significant differences between media MEM and F99 as well as between media F99 and SFM (p<0.05) (Fig 3). FCS supplementation in all three media tested in this study had no significant influence on the necrotic areas in the endothelial cell layer ( $p$ $>0.05$ ) (Fig 3).

With respect to the condition of the keratocytes, after the culture period of 3 weeks we compared the amount and the viability of these cells in three different corneal layers: the anterior stroma underlying the epithelium, the central stroma, and the posterior stroma next to Descemet membrane. The corneas cultured in Endothelial-SFM exhibited the highest overall number of keratocytes especially when cultured without serum supplementation. The number of keratocytes was relatively low only in the anterior part of the stroma with a high portion of dead cells. The morphology of the keratocytes was comparable with that observed

Table 1 Endothelial cell densities at the beginning of organ culture and after 1, 2, and 3 weeks of culture in medium $M E M, F 99$, and endothelial-SFM (SFM) with or without serum supplementation. Indicated are the means (SD) of the corneal endothelial cell densities of seven cornea

\begin{tabular}{lllll}
\hline Medium & Before organ culture & After 7 days of organ culture & After 14 days of culture & After 21 days of culture \\
\hline MEM without FCS & $2360(470)$ & $1920(350)$ & $1880(250)$ & $1370(630)$ \\
MEM + 2\% FCS & $2190(590)$ & $1960(450)$ & $1590(430)$ & $1590(470)$ \\
F99 without FCS & $2390(310)$ & $2020(340)$ & $1970(330)$ & $1770(370)$ \\
F99 + 2\% FCS & $2260(380)$ & $1790(520)$ & $1720(420)$ & $1640(470)$ \\
SFM without FCS & $2600(210)$ & $1960(420)$ & $2040(280)$ & $1940(200)$ \\
SFM + 2\% FCS & $2510(340)$ & $1930(340)$ & $2030(230)$ & $1840(380)$ \\
\hline
\end{tabular}




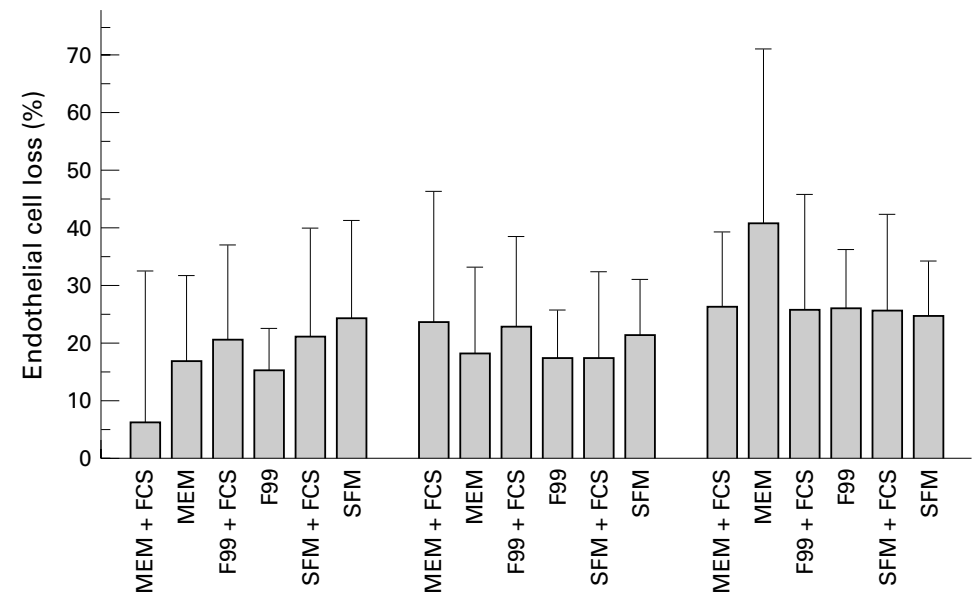

After 1 week

After 2 weeks

After 3 weeks

Figure 2 Endothelial cell loss of donor corneas during organ culture in medium MEM, F99, and Endothelial-SFM (SFM) with or without supplementation of $2 \%$ FCS. Cell densities were determined at the beginning of organ culture and then every week. Indicated are the means (SD) for the decrease in endothelial cell density determined from seven corneas.

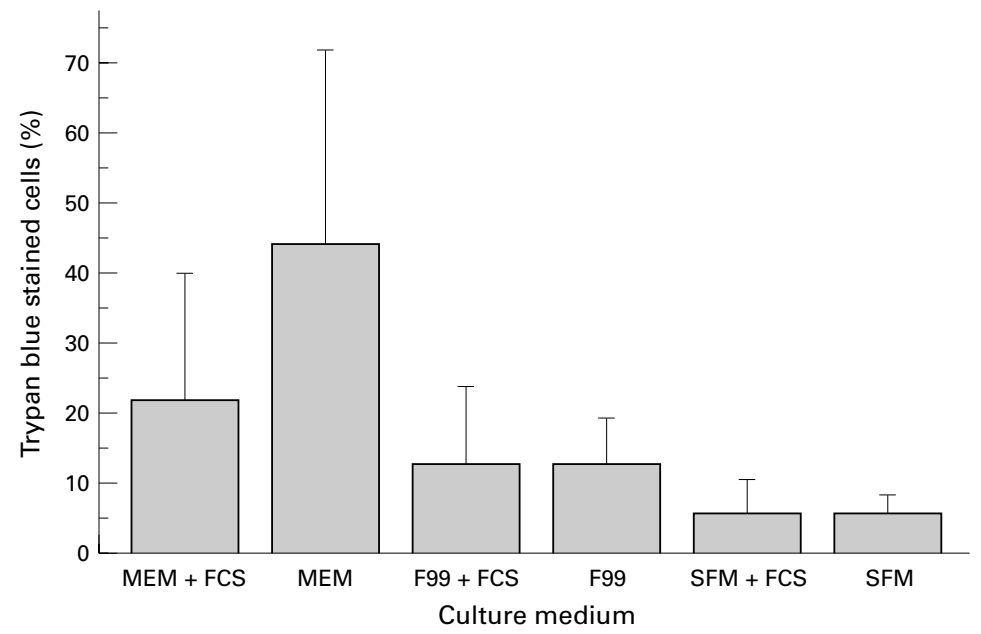

Figure 3 Analysis of damaged endothelium after 3 weeks of organ culture by means of trypan blue staining. Indicated are the means (SD) of the percentage of blue stained endothelial cells from seven corneas cultivated in the indicated medium with or without $2 \%$ FCS supplementation. Statistical analysis revealed the following results:

MEM with FCS versus SFM with FCS $M E M$ without FCS versus SFM without FCS $M E M$ with FCS versus F99 with FCS

$M E M$ without FCS versus F99 without FCS F99 with FCS versus SFM with FCS

F99 without FCS versus SFM without FCS

SFM with FCS versus SFM without FCS

$M E M$ with FCS versus $M E M$ without FCS

F99 with FCS versus $F 99$ without FCS

$\begin{array}{ll}\text { p Value } & \text { Significance } \\ 0.036 & \text { Yes } \\ 0.003 & \text { Yes } \\ 0.260 & \text { No } \\ 0.012 & \text { Yes } \\ 0.132 & \text { No } \\ 0.018 & \text { Yes } \\ 0.943 & \text { No } \\ 0.100 & \text { No } \\ 1.000 & \text { No }\end{array}$

in the other corneas but the staining was not as intensive as that found after culture in MEM or F99.

The number of keratocytes in the anterior stroma was highest in corneas cultivated in MEM compared with the other corneas. The cells exhibited a large variation in shape and morphology. The keratocytes were less frequent in the central part of the cornea where they exhibited a pyramidal shape with some network forming prolongations. Round or spindle-like keratocytes were found in the posterior stroma in a relatively low concentration. In some corneas cultivated in MEM without serum supplementation, it was not possible to identify the different layers of the stroma with their typical keratocytes. Moreover, the typical parallel arrangement of keratocytes in the central part of the stroma was no longer detectable.

The lowest overall number of keratocytes was observed after the culture in medium F99. Supplementation of the medium with FCS resulted in only a slight increase in the number of keratocytes. The morphology of the keratocytes within the different parts of the stroma was comparable with those of corneas cultivated in medium MEM. No incidence for dead keratocytes was found in the posterior part of the cornea.

\section{Discussion}

Bovine serum is a complex mixture of different substances, some of which are as yet undetermined. Furthermore, variability in the composition of the serum can occur from lot to lot. In addition, it is known that even minor changes in the culture medium can have an influence on the quality of the donor corneas. Therefore, it is desirable to have a well defined and consistent medium suitable for the preservation of human donor corneas.

Serum may be a possible source of prions, which are thought to induce a new form of Creutzfeldt-Jakob disease (CJD). A case of growth hormone related CJD has been reported. ${ }^{17}$ Furthermore, evidence exists for the transmission of CJD via corneal transplants, ${ }^{18} 19$ but in these cases corneas were derived from donors suffering from CJD. Although there is no evidence of transmission of prion protein by use of serum supplementation in organ culture medium, the use of a serum free culture medium would exclude any risk of transmission of infection via bovine serum.

In the first part of this study we chose to investigate the effect of different media on isolated human corneal endothelial cells because the endothelium represents the most important cell layer of the cornea. We used immortalised human corneal endothelial cells ${ }^{11}$ which exhibit morphological and functional properties comparable with normal human corneal endothelial cells ${ }^{2021}$ to avoid possible variations caused by different properties of primary cell cultures established from various donors.

Stimulation of proliferation of cultured human corneal endothelial cells was comparable in the case of all three mentioned media when serum supplementation was used. This finding corresponds to the results of our previous study where proliferation of corneal endothelial cells was analysed after 18 day of culture in 11 different basal media, ${ }^{10}$ a study in which higher serum levels were tested on primary human corneal endothelial cells. Long term culture of the cells without serum supplementation was only possible in the Endothelial-SFM medium. This result indicates that Endothelial-SFM medium contains all substances sufficient to maintain viability of cultured human corneal endothelial cells and 
even to provoke their proliferation. The comparatively low proliferation activity within the first week of culture in Endothelial-SFM medium may be due to adaptation.

In the second part of the study we incubated donor corneas in the different culture media. The results demonstrated that in the conventionally used MEM medium, serum supplementation was obligatory as had been shown by the development of large areas of necrosis within the endothelium revealed by trepan blue staining. Trypan blue staining has been shown to be a simple method to determine membrane integrity of corneal endothelial cells ${ }^{16}$ and is routinely used in some cornea banks. ${ }^{8}$ The dye is incorporated only by damaged cells and leads to intensive blue staining.

The highest number of damaged endothelial cells was found in MEM medium without serum. Serum supplementation led to the reduction of the endothelial damage but the value remained higher than in corneas incubated in medium F99. The best results were found in Endothelial-SFM medium. Viability testing is more readily apparent than the determination of endothelial cell density, because damaged cells can be identified and monitored long before they die, leading to the decrease in endothelial cell density.

The density of keratocytes within the stroma decreases during the culture in MEM but their proliferative capacity is maintained..$^{22}$ In freshly prepared corneas Poole et $a l^{4}$ described three different types of keratocytes: in the anterior stroma small keratocytes forming a three dimensional network; in the central stroma pyramidal cells with short prolongations by which only neighbouring cells of the same plane are connected; and in the posterior stroma, large keratocytes with short prolongations forming a three dimensional network. In this study, viable keratocytes were found in all layers of all corneas in all three media after 3 weeks of cultivation. The main difference after organ culture in the three mentioned media concerned the keratocyte density in the central part of the stroma. The highest keratocyte density was found in corneas cultivated in Endothelial-SFM medium with or without serum supplementation. This may indicate that Endothelial-SFM satisfies the nutritional requirements of the endothelium as well as of the keratocytes during organ culture. The lowest density was found in the central stroma of corneas when cultivated in MEM medium. This indicates that a so called "minimal" basal medium is not sufficient for cornea banking.

On the other hand F99 medium which was developed for optimised culture of human corneal endothelial cells in vitro ${ }^{9}$ did not give the best results in the preservation of whole corneas. This may be due to the fact that F99 was designed to provoke corneal endothelial cell proliferation in vitro, whereas proliferation of these cells in situ does not occur. Furthermore the presence of keratocytes and the stroma in the organ culture may influence the nutrient demand of the endothelial cells in comparison with pure cultures of corneal endothelial cells.
In a previous study we have shown, that serum supplemented Endothelial-SFM is a promising basal medium for organ culture of human donor corneas. ${ }^{10}$ In this study we demonstrate for the first time that this medium is also suitable for cornea preservation without serum supplementation. The use of Endothelial-SFM can exclude any variability in medium composition and also minimise the risk of infection resulting from the supplementation of serum.

1 Bourne WM, Doughman DJ, Lindstrom RL, et al. Increased endothelial cell loss after transplantation of corneas preserved by a modified organ-culture technique. Ophthalpreserved by a modified
mology 1984;91:285-9.

2 Bourne WM. Endothelial cell survival on transplanted human corneas preserved at $4^{\circ} \mathrm{C}$ in $2.5 \%$ chondroitin sulfate for one to thirteen days. Am $\mathcal{F}$ Ophthalmol 1986;102: 382-6.

3 Pels E, Schuchard Y. Organ culture preservation of human corneas. Doc Ophthalmol 1983;56:147-53.

4 Schultz G, Cipolla L, Whitehouse A, et al. Growth factors and corneal endothelial cells: III. Stimulation of adult human corneal endothelial cell mitosis in vitro by defined mitogenic agents. Cornea 1992;11:20-7.

5 Barisani-Asenbauer T, Kaminski S, Schuster E, et al. Impact of growth factors on morphometric corneal endothelial cell parameters and cell density in culture-preserved human corneas. Cornea 1997;16:537-40.

6 Engelmann K, Friedl P. Growth of human corneal endothelial cells in a serum-reduced medium. Cornea 1995;14:62-

7 Engelmann K, Sobottka Ventura A, Drexler D, et al. A sensitive method for testing the quality of organ culture media of individual medium components in a cornea bank. Graefes Arch Clin Exp Ophthalmol 1998;236:312-19.

8 European Eye Bank Association Directory. 8th ed. Århus, Denmark, January 2000.

9 Engelmann K, Friedl P. Optimization of culture conditions for human corneal endothelial cells. In Vitro Cell Dev Biol 1989;25:1065-72.

10 Møller-Pedersen T, Hartmann U, Ehlers N, et al. Evaluation of potential organ culture media for eye banking using a human corneal endothelial cell growth assay. Graefes Arch Clin Exp Ophthalmol (in press).

11 Bednarz J, Teifel $\mathrm{M}$, Friedl $\mathrm{P}$, et al. Immortalization of human corneal endothelial cells using electroporation protokol optimized for human corneal endothelial and human retinal pigment epithelial cells. Acta Ophthalmol Scand 2000;78:130-6

12 Litzkas P, Jha KK, Ozer H L. Efficient transfer of cloned DNA into human diploid cells: Protoplast fusion in suspension. Mol Cell Biol 1984;4:2549-52.

13 Engelmann K, Böhnke M, Friedl P. Isolation and long-term cultivation of human corneal endothelial cells. Invest Ophthalmol Vis Sci 1988;29:1656-62.

14 Engelmann K, Böhnke M. Human corneal endothelial cells in long-term cultures: the influence of conditions for isolation, selective and normal growth, and the extracellular matrix on proliferation and morphology. Chibret Int $\mathcal{F} O \mathrm{ph}$ thalmol 1990;7:3-12.

15 Böhnke M. Corneal preservation in organ culture. Curr Opin Ophthalmol 1991:2:432-42.

16 Singh G, Böhnke M, von Domarus D, et al. Vital staining of corneal endothelium. Cornea 1985;4:80-91.

17 Markus HS, Duchen LW, Parkin EM, et al. CreutzfeldtJakob disease in recipients of human growth hormone in the United Kingdom: A clinical and radiographic study. $Q$ f Med 1992;297:43-51.

18 Duffy P, Wolf J, Collins G, et al. Possible person-to-person transmission of Creutzfeldt-Jacob disease. $N$ Engl F Med 1974;290:692-3.

19 Heckmann JG, Lang CJG, Petruch F, et al. Transmission of Creutzfeld-Jakob disease via a corneal transplant. $\mathcal{F}$ Neurol Neurosurg Psychiatry 1997;63:388-90.

20 Aboalchamat B, Engelmann K, Böhnke M, et al. Morphological and functional analysis of immortalized human corneal endothelial cells after transplantation. Exp Eye Res 1999:69:547-53.

21 Bednarz J, Aboalchamat B, Engelmann K. Characterization of immortalized human corneal endothelial cells. Invest Ophthalmol Vis Sci (Abstract) 2000;41:S448.

22 Salla S, Redbrake C, Becker J, et al. Remarks on the vitality of the human cornea after organ culture. Cornea 1995;14: 502-8.

23 Møller-Pedersen T, Møller HJ. Viability of human corneal keratocytes during organ culture. Acta Ophthalmol Scand 1996;74:449-55

24 Poole CA, Brookes NH, Clover GM. Keratocyte network visualised in the living cornea using vital dyes. Cell Sci 1993;106:685-92. 